\title{
Marcadores de estresse oxidativo e dano muscular em pacientes com DPOC submetidos a um programa de reabilitação pulmonar
}

\author{
Oxidative stress and muscle damage markers in COPD patients in a pulmonary recovery \\ program
}
Marcadores de estrés oxidativo y daño muscular en pacientes con EPOC en un programa de recuperación pulmonar

Shaumin Vasconcelos $\mathrm{Wu}^{1 *}$, Amanda Caroline Lobato Dias ${ }^{1}$, Dayane Cereja Ferreira da Silva ${ }^{1}$, Ingrid Ferreira Santos ${ }^{1}$, William Wallace Cordeiro dos Santos ${ }^{1}$, Renata Cunha Silva ${ }^{1}$, Pedro luri Castro da Silva ${ }^{1}$, Jofre Jacob da Silva Freitas ${ }^{1}$, Luiz Fábio Magno Falcão ${ }^{1}$, Valéria Marques Ferreira Normando ${ }^{1}$.

\section{RESUMO}

Objetivo: Este artigo buscou analisar o comportamento do estresse oxidativo e a expressão sérica da creatina quinase em portadores de doença pulmonar obstrutiva crônica participantes de um programa de reabilitação pulmonar. Métodos: Trata-se de um ensaio clínico analítico, longitudinal, prospectivo e quantitativo, aprovado pelo comitê de ética em pesquisa com seres humanos (n-1.965.332). Seis voluntários, com média de idade 67,8 , realizaram a espirometria e a análise sanguínea para leitura da creatina quinase e do estresse oxidativo, antes da $1^{\text {a }}$ sessão (T0) e após a $20^{a}$ sessão (T20) do protocolo de Reabilitação Pulmonar. Utilizou-se o teste análise de variância (ANOVA) para comparar as médias entre os dois momentos de testagem por meio do software BioEstat 5.0 com $p \leq 0,05$. Resultados: Houve resposta significativa dos marcadores de proteólise muscular por meio da quantificação da creatina quinase isoenzima MB (CK-MB; $p=0.026$ ) e creatina quinase total (CK-NAC; $p=0.017$ ) e não significativa para a análise bioquímica do estresse oxidativo (Catalase; $p=$ 0.764) e (Peroxidase lipídica; $p=0.584$ ) ao comparar os tempos T0 e T20. Conclusão: Ocorreu um impacto positivo sob a expressão sérica da creatina quinase em portadores de doença pulmonar obstrutiva crônica durante a condução de um protocolo de reabilitação pulmonar.

Palavras-chave: Doença pulmonar obstrutiva crônica, Creatina quinase, Estresse oxidativo, Fisioterapia.

\begin{abstract}
Objective: This article sought to analyze the behavior of oxidative stress and the serum expression of creatine kinase in patients with chronic obstructive pulmonary disease participating in a pulmonary rehabilitation program. Methods: This is a study analytical, longitudinal, prospective and quantitative clinical trial, approved by the Research Ethics Committee (n-1.965.332). Six volunteers, with a mean age of 67.8, underwent spirometry and blood analysis to read creatine kinase and oxidative stress, before the 1st session (T0) and after the 20th session (T20) of the Pulmonary Rehabilitation protocol. The analysis of variance test (ANOVA) was used to compare the means between the two testing moments using the BioEstat 5.0 software with $p$ $\leq 0.05$. Results: There was a significant response of muscle proteolysis markers (CK-MB; $p=0.026$ ) and (CKNAC; $p=0.017$ ) and not significant for the biochemical analysis of oxidative stress (Catalase; $p=0.764$ ) and (Lipid peroxidase; $p=0.584$ ) when comparing the times T0 and T20. Conclusion: There was a positive impact on serum creatine kinase expression in patients with chronic obstructive pulmonary disease while conducting a pulmonary rehabilitation protocol. impact on serum creatine kinase expression in patients with chronic obstructive pulmonary disease while conducting a pulmonary rehabilitation protocol.
\end{abstract}

Keywords: Chronic obstructive pulmonary disease, Creatine kinase, Oxidative stress, Physiotherapy.

1 Universidade do Estado do Pará (UEPA), Belém - PA. *E-mail: shaumin.wu@gmail.com

Financiamento pelo Conselho Nacional de Desenvolvimento Científico e Tecnológico (CNPQ)

SUBMETIDO EM: $3 / 2021$

ACEITO EM: 3/2021

PUBLICADO EM: 5/2021 


\section{RESUMEN}

Objetivo: Este artículo buscaba analizar el comportamiento del estrés oxidativo y la expresión sérica de creatina quinasa en pacientes con enfermedad pulmonar obstructiva crónica que participan en un programa de rehabilitación pulmonar. Métodos: Este es un estudio cuyo ensayo clínico analítico, longitudinal, prospectivo y cuantitativo, aprobado por el Comité de Ética en Investigación (no 1.965.332). Seis voluntarios, con una edad media de 67.8 años, se sometieron a espirometría y análisis de sangre para leer creatina quinasa y estrés oxidativo, antes de la primera sesión (T0) y después de la vigésima sesión (T20) del protocolo de rehabilitación pulmonar. La prueba de análisis de varianza (ANOVA) se usó para comparar los promedios entre los dos momentos de prueba usando el software BioEstat 5.0 con $p \leq 0.05$. Resultados: respuesta significativa de marcadores de proteólisis muscular (CK-MB; $p=0.026$ ) y (CK-NAC; $p=0.017$ ) y no significativa para el análisis bioquímico del estrés oxidativo (Catalasa; $p=0.764$ ) y (peroxidasa lipídica; $p=$ 0.584) al comparar los tiempos T0 y T20. Conclusión: Así, observa-se Impactó positivo en la expresión de creatina quinasa sérica en pacientes con enfermedad pulmonar obstructiva crónica mientras se realiza un protocolo de rehabilitación pulmonar.

Palabras clave: Enfermedad pulmonar obstructiva crónica, Creatina quinasa, Estrés oxidativo, Fisioterapia.

\section{INTRODUÇÃO}

A Doença Pulmonar Obstrutiva Crônica (DPOC) representa a terceira causa de morte entre as doenças crônicas não transmissíveis no Brasil, e atinge 64 milhões de pessoas, sendo em 2015, gerados mais de 3,2 milhões de mortes em todo o mundo. Em 2015 a DPOC foi responsável pela morte de mais de 3,2 milhões de pessoas, num total de 64 milhões de portadores da doença. A nível global, estima-se que ela estará entre as três principais causas de morte até 2030 (MERCK SHARP AND DOHME (MSD) 2018). É caracterizada pela obstrução crônica e progressiva do fluxo expiratório, associada a uma resposta inflamatória anormal das vias aéreas e do parênquima pulmonar a partículas e gases tóxicos, e sua prevalência varia de acordo com a região e o índice de tabagismo (RABAHI MF, 2013; ZOPPI D e FILHO ABA, 2018).

Entre as manifestações locais, destaca-se a hiperinsuflação pulmonar, que altera os movimentos ventilatórios realizados pelas costelas, a inflamação das vias aéreas e a destruição do parênquima pulmonar. As manifestações clínicas e funcionais da DPOC resultam de danos pulmonares provocados por um conjunto de mecanismos, incluindo a inflamação, o desequilíbrio do sistema protease-antiprotease, a apoptose e, principalmente, o estresse oxidativo (BMJ, 2020).

Investigações clínicas sugerem que o estresse oxidativo desempenha um papel importante na gênese, desenvolvimento e manutenção do quadro clínico da DPOC, implicando em efeitos locais e sistêmicos da doença (TAVILANI H, et al., 2012).

Inicialmente, na DPOC, o estresse oxidativo mantém-se restrito ao tecido pulmonar devido ao aumento de células inflamatórias. Estas se mantêm ativadas ocasionando progressiva destruição do parênquima pulmonar, gerando o aumento da formação das moléculas oxidantes (CAVALCANTE AGM e BRUIN PFC, 2009). Essas moléculas podem atuar aumentando a secreção de muco e a permeabilidade da membrana capilar, levando a broncoconstrição. Esses fatores são os desencadeadores dos sintomas iniciais característico da DPOC como tosse, secreção e dispnéia crônica ao esforço (GONÇALVES APFG e COUTO $V F, 2021)$.

Os pulmões não são os únicos órgãos atingidos pelo estresse oxidativo na DPOC (CAMPOS HS, 2006). Quando ocorre a expansão do estresse oxidativo para fora do tecido pulmonar, via corrente sanguínea, outros sistemas e órgãos são atingidos. Isso ocasiona os efeitos extrapulmonares ou sistêmicos da doença (FISCHER BM, et.al., 2011). Estudos têm demonstrado que níveis elevados de oxidantes periféricos podem ser consequência da diminuição da capacidade antioxidante nesses pacientes (CAMPOS MTG, LEME FOP, 2018). Em nível sistêmico, tais biomarcadores podem acarretar os principais efeitos extrapulmonares da 
doença. Entre esses efeitos estão a fraqueza muscular periférica e a intolerância ao exercício. Como também suas principais comorbidades: doenças cardíacas, osteoporose, desnutrição e depressão (MOURA MMMC, et al., 2012).

Além disso, o desgaste da proteína Creatina Quinase (CK) é considerada como evidência de desgaste muscular, uma vez que normalmente esta enzima não é capaz de atravessar a membrana celular. Considerase que o desgaste desta enzima através dos vasos refletiria alterações importantes ocorridas na estrutura das membranas, ou seja, alteração da fibra muscular (NUNES E, CURTY VM, 2012).

Nesse contexto, os programas de reabilitação pulmonar (RP), baseados em exercícios físicos, buscam o foco na diminuição da dispnéia e melhora da endurance (SPROUT MA, et al., 2013). Atualmente sabe-se que a reabilitação pulmonar alivia a dispneia e a fadiga, melhora a função emocional e aumenta a sensação de controle que os indivíduos têm sobre sua condição. Essas melhorias são moderadamente substanciais e clinicamente significativas. Portanto, a reabilitação serve como um componente essencial do manejo da DPOC e é benéfica na melhoria da qualidade de vida relacionada à saúde e da capacidade de exercício no que se refere ao bem estar biopsicossocial (FIORENTINO G, et al., 2020).

Nesse contexto, a Caminhada Nórdica vem sendo apontada como importante recurso complementar à reabilitação pulmonar. Essa terapia começou a ser realizada na Europa, e caracteriza-se por utilizar os bastões nórdicos como auxílio da caminhada, auxiliando na segurança e propulsão dos pacientes, é uma forma de tratamento para pacientes portadores de enfermidades do sistema cardiorrespiratório e sabe-se que pode influenciar diretamente na qualidade de vida, principalmente em curto prazo (SKÓRKOWSKATELICHOWSKA K, et al., 2016; RUGBJERG M, et al., 2015).

No entanto, poucos estudos visam investigar os efeitos do exercício sobre parâmetros inflamatórios e metabólicos em pacientes com DPOC (SILVA BSA, et al., 2018). Desta forma, torna-se necessária a investigação de estudos que visem compreender estratégias para minimizar tais efeitos, não somente avaliando os efeitos clínicos como o comportamento dos parâmetros bioquímicos. Por isso, a presente pesquisa teve como objetivo analisar o comportamento do estresse oxidativo e a expressão sérica da CK, em portadores de doença pulmonar obstrutiva crônica, participantes de um programa de reabilitação pulmonar.

\section{MÉTODOS}

\section{Desenho do estudo}

Trata-se de um ensaio clínico analítico, longitudinal, prospectivo e quantitativo, de escala local. Todos os procedimentos foram aprovados pelo comitê de ética em pesquisa com seres humanos da Universidade do Estado do Pará (UEPA), sob número de parecer 1.965.332, CAAE: 44880115.3.0000.5174. Além disso, foi uma pesquisa aprovada como projeto de Iniciação Científica com verba do Conselho Nacional de Desenvolvimento Científico e Tecnológico (CNPQ).

\section{Sujeitos da pesquisa}

Foram incluídos no estudo voluntários idosos, sem alterações cognitivas de ambos os gêneros, de qualquer etnia, faixa etária entre 60 a 80 anos e com diagnóstico clínico e espirométrico de disfunção obstrutiva leve segundo a classificação da Global initiative for chronic Obstructive Lung Disease (GOLD), clinicamente estáveis, sem exacerbação da doença, ou seja, aumento e/ou mudança no aspecto da secreção respiratória, tosse, fadiga e aumento da dispnéia. Foram excluídos voluntários que apresentavam outras doenças metabólicas e comorbidades graves tais como: cardiopatia e hipertensão arterial não controlada; tabagista ainda ativo ou que cessou o hábito há menos de um ano do início do estudo; bem como, aqueles que estiveram internados pelo menos seis meses antes do estudo.

\section{Teste de função pulmonar}

Todos os voluntários da pesquisa foram submetidos a uma avaliação espirométrica, sendo realizada na Clínica Pulmocenter, por meio do SPIROM2 Spiromatic $\AA^{\circledR}$ (Engelógica-Rio de Janeiro-RJ), que consistiu em 
mensurar o volume de fluxo, incluindo medições de FEV1 e FVC, com o maior valor de pelo menos três medições realizadas corretamente sendo usadas para análise. Os valores obtidos foram expressos como uma porcentagem do valor de referência.

\section{Procedimentos experimentais}

\section{Coleta de sangue ou de amostras biológicas}

Todos os participantes foram submetidos a uma coleta de $10 \mathrm{ml}$ de sangue com heparina de uma veia antecubital, antes e após o programa de reabilitação pulmonar. A coleta era realizada no período matutino, em jejum de 12 horas, sendo realizada no Laboratório de Análises Clínicas da Universidade do Estado do Pará - UEPA. O sangue foi centrifugado ( $1500 \mathrm{rpm}, 15$ minutos a $4^{\circ} \mathrm{C}$ ) para a separação do plasma que foi armazenado a $-80^{\circ} \mathrm{C}$ até a análise do estresse oxidativo. A análise do estresse oxidativo foi realizada no Laboratório de Morfofisiologia Aplicada à Saúde da UEPA.

\section{Programa de Reabilitação Pulmonar}

Todos os voluntários realizaram o protocolo de reabilitação pulmonar (PRP), no total de 20 sessões, com duração em média de 60 minutos, no período matutino, três vezes por semana (SEVERO VG e RECH VV, 2006). Cada sessão do PRP era composta por exercícios de alongamentos global, exercícios intervalados (reeducação diafragmática, exercícios metabólicos e circuito de atividades de vida diária (AVDs), treinamento de membros superiores e inferiores, treino de resistência, com caminhada nórdica e alongamento e relaxamento muscular finais. O PRP foi desenvolvido no setor de fisioterapia respiratória da Unidade de Ensino e Assistência de Fisioterapia e Terapia Ocupacional (UEAFTO) da UEPA.

\section{Creatina quinase}

Para a análise da creatina quinase (CK), o protocolo foi baseado no Método UV otimizado (IFCC) para a determinação de CK em soro ou plasma - creatina quinase total (CK-NAC). O soro foi recolhido para análise da CK. As condições para reação ocorrer foram em equipamento automatizado de bioquímica em comprimento de onda: $340 \mathrm{~nm} \mathrm{Hg} 334$ e temperatura da reação: $37^{\circ} \mathrm{C}$, com objetivo de melhorar a absorção. A leitura do material foi realizada em dois momentos. Primeiramente, a amostra foi mantida em uma cubeta de em temperatura de $30^{\circ}$ à $37^{\circ} \mathrm{C}$. Foi adicionado $1 \mathrm{ml}$ de reagente único, que é formado pela mistura de 5 partes de Reagente A com 1 parte de Reagente B (ex. $5 \mathrm{ml}$ Reagente A 1 ml Reagente B). Sendo tais reagentes compostos por: reagente $A$ : solução de tampão imidazol e reagente $B$ : solução de componentes contendo creatina fosfato e componentes reativos. A análise foi realizada no Laboratório de Análises Clínicas da Universidade do Estado do Pará.

\section{Substâncias que regem ao ácido tiobarbitúrico (TBARS)}

A mensuração da Peroxidação Lipídica foi analisada a partir da concentração de TBARS e mensurada conforme o método descrito por Winterbourn, que consiste na reação colorimétrica entre produtos da peroxidação lipídica e o ácido tiobarbitúrico a $1 \%$, a temperatura que varia entre 90 a $100^{\circ} \mathrm{C}$. Foi adicionado dentro de um microtubo de polipropileno $20 \mu \mathrm{L}$ de BHT (solução etanólica a $2 \%$ ), $200 \mu \mathrm{L} \mathrm{HCl} \mathrm{a} 25 \%, 200 \mu \mathrm{L}$ de ácido tiobarbitúrico a $1 \%$ (dissolvido em solução aquosa com $0.05 \mathrm{~N} \mathrm{NaOH}$ ) e $200 \mu \mathrm{L}$ do plasma. As amostras foram incubadas em banho seco entre $90^{\circ}$ a $100^{\circ} \mathrm{C}$ durante 15 minutos, e após esse período, foram colocadas em gelo para esfriar e foi adicionado $618 \mu \mathrm{L}$ de butanol em cada amostra, em seguida, centrifugadas por 2 minutos a 4000 RPM. Após retirado $200 \mu \mathrm{L}$ do sobrenadante e analisados em duplicata na placa de 96 poços e lido no espectrofotômetro a $532 \mathrm{~nm}$. A concentração de TBARS é expressa em nM/mg de proteína.

\section{Catalase}

A atividade da enzima catalase foi determinada segundo o método descrito por Aebi $H$ (1984), que quantifica a velocidade de decomposição do peróxido de hidrogênio, em $240 \mathrm{~nm}$ durante 6 minutos, pela enzima presente na amostra. Utiliza-se a solução de peróxido de hidrogênio (H2O2) $10 \mathrm{mM}$ em tampão fosfato $\mathrm{mM} \mathrm{pH} \mathrm{7,0} \mathrm{preparada} \mathrm{e} \mathrm{titulada} \mathrm{no} \mathrm{dia} \mathrm{da} \mathrm{análise.} \mathrm{Para} \mathrm{isso,} \mathrm{foi} \mathrm{adicionado} 2 \mathrm{~mL}$ desta solução na cubeta, com acréscimo de $20 \mu \mathrm{L}$ da amostra, em seguida, foi feita a leitura da queda da absorbância. Os valores foram expressos em $\mathrm{mmol} / \mathrm{min}-1 \mathrm{~mL}$. 


\section{Determinação de proteínas}

O método de Bradford foi utilizado para mensurar a concentração de proteína total por meio da espectrofotometria, que se baseia na reação colorimétrica entre a proteína no interior da amostra e a solução de Bradford (Blue Coomassie 0,01\%, etanol 4,75\%, ácido fosfórico 8,5\%). Foram adicionados $20 \mu$ do plasma em $1 \mathrm{~mL}$ da solução de Bradford que foi homogeneizada e levada para incubar ao abrigo da luz durante 5 minutos a $4^{\circ} \mathrm{C}$. Após o processo, foi retirado $200 \mu \mathrm{L}$ da mistura e analisados em duplicatas na placa de 96 poços e lidos a $595 \mathrm{~nm}$ no espectrofotômetro. A concentração de proteína da amostra foi estimada por equivalência de albumina (absorbância 0,66 a $280 \mathrm{~nm}$ ). Os resultados foram expressos em mg/mL.

\section{Análise estatística}

Os dados foram tabulados em uma planilha do Microsoft Excel e para análise dos resultados, foi utilizado o software BioEstat 5.0 com o Teste t de amostra pareada para comparar as médias entre os dois momentos de testagem (antes e no fim do tratamento). Foi adotado um nível de significância para as inferências estatísticas de $\mathrm{p} \leq 0,05$.

\section{RESULTADOS}

Inicialmente foram convidados 30 indivíduos para participarem da pesquisa. Após triagem por meio dos critérios de inclusão e exclusão, finalizaram o protocolo de reabilitação pulmonar, 6 indivíduos como demonstra a Figura 1.

Figura 1 - Fluxograma dos participantes do projeto.

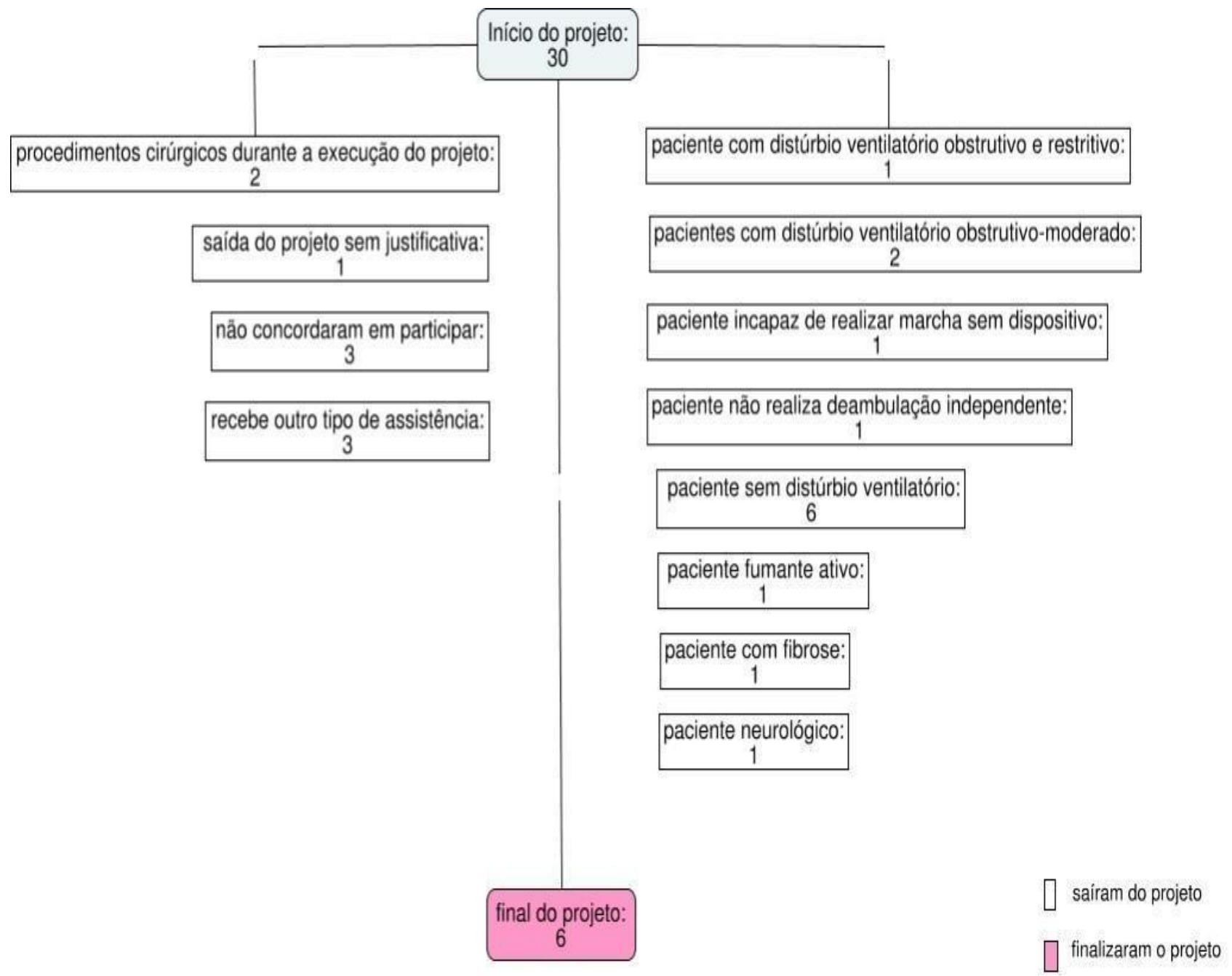

Fonte: Wu SV, et al., 2021. 
A respeito dos dados sócio demográficos, quanto a caracterização da amostra foi composta de 6 indivíduos incluídos no estudo com diagnóstico clínico e espirométrico espirométrico de DPOC. Destes, $3(50 \%)$ eram homens e $3(50 \%)$ eram mulheres. Os valores médios obtidos dos pacientes com DPOC para idade foram de $67,8 \pm 7,19$ anos. A predominância dos participantes com estado civil casado e com escolaridade na sua maioria com ensino fundamental. Os dados sociodemográficos dos indivíduos estão descritos na Tabela 1.

Tabela 1 - Características gerais dos indivíduos incluídos no estudo.

\begin{tabular}{cc}
\hline Variáveis & Indivíduos \\
\hline Idade (anos) & $67,8 \pm 7,19$ \\
\hline Sexo (M/F) & $03 /$ mar \\
\hline Estado Civil & \\
\hline Casado & $83,33 \%$ \\
Divorciado & $16,66 \%$ \\
\hline Escolaridade & \\
\hline Ensino fundamental & $66,33 \%$ \\
Ensino médio & $33,33 \%$ \\
\hline
\end{tabular}

Legenda: Valores expressos em média \pm Desvio Padrão (DP).

Fonte: Wu SV, et al., 2021.

O dano muscular foi avaliado por meio dos valores de concentração sérica de CK-NAC (figura 02, A) e CK-MB (figura 02, B) nos indivíduos com DPOC pré e pós aplicação do PRP. Observou-se uma diminuição na concentração dessas proteínas, estatisticamente significante CK-NAC (Pré-PRP: 155,4 $\pm 7,29$ U/l e PósPRP: $121,0 \pm 55,72 \mathrm{U} / \mathrm{l}$ com p=0.004) e CK-MB (Pré-PRP 24,0 $\pm 8,72 \mathrm{U} / \mathrm{l}$ e Pós-PRP 17,20 \pm 5,45 U/l com $\mathrm{p}=0.002$ ), a aplicação do protocolo de reabilitação pulmonar, como demonstra a Figura 2.

Figura 2 - Níveis de dano muscular em indivíduos com DPOC antes (pré) e após (pós) o programa de reabilitação pulmonar (PRP).

A

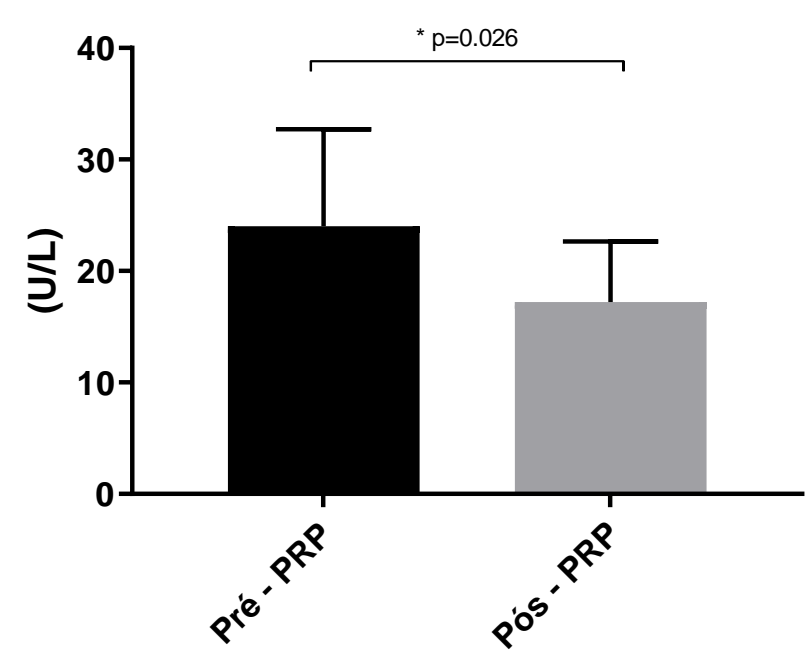

B CK-NAC

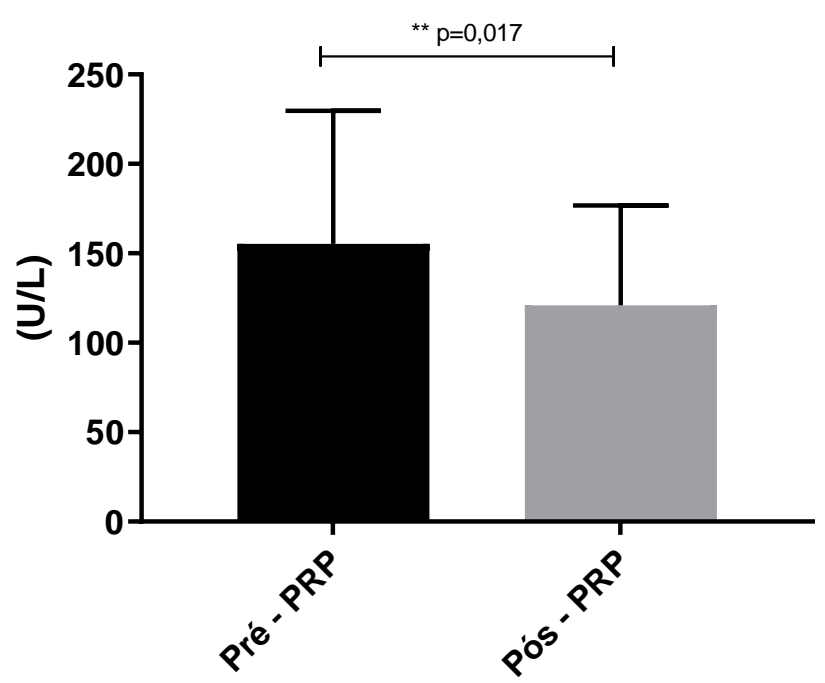

Legenda: CK-MB: níveis sericos de creatina quinase isoenzima MB. CK-NAC: níveis séricos de creatina quinase total. Pré-PRP: pré protocolo de reabilitação pulmonar. Pós-PRP: pós protocolo de reabilitação pulmonar. Valores expressos em média \pm Desvio Padrão (DP).

Fonte: Wu SV, et al., 2021.

A análise do estresse oxidativo foi realizada pela mensuração das substâncias reativas ao ácido tiobarbitúrico (TBARS) e pela concentração da enzima antioxidante catalase. Quanto aos níveis de TBARS observou-se que não houve diferença estatisticamente significante pré $(0.505 \pm 0.115 \mathrm{mg} / \mathrm{ml})$ e pós $(0.480 \pm$ 
$0.225 \mathrm{mg} / \mathrm{ml}), \mathrm{p}>0,05$, a aplicação do PRP. Assim como, não houve diferença estatisticamente significante ( $p>0,05)$ para os valores de catalase pré $(0.488 \pm 0.370)$ e após $(0.565 \pm 0.444)$ a aplicação do PRP, como demonstram as Figuras 3 e 4, respectivamente.

Figura 3 - Níveis de substâncias que reagem ao ácido tiobarbitúrico (TBARS) em indivíduos com DPOC antes (pré) e após (pós) o programa de reabilitação pulmonar (PRP).

TBARS

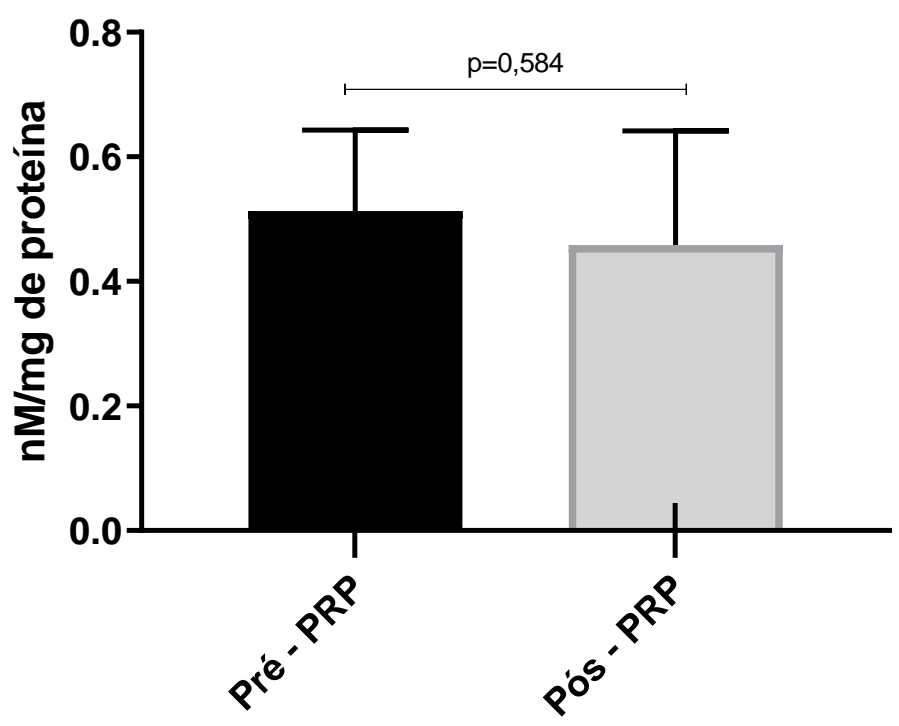

Legenda: TBARS: substâncias que reagem ao ácido tiobarbitúrico. Pré-PRP: pré protocolo de reabilitação pulmonar. Pós-PRP: pós protocolo de reabilitação pulmonar.

Valores expressos em média \pm Desvio Padrão (DP).

Fonte: Wu SV, et al., 2021.

Figura 4 - Concentração de catalase em indivíduos com DPOC antes (pré) e após (pós) o programa de reabilitação pulmonar (PRP).

\section{Catalase}

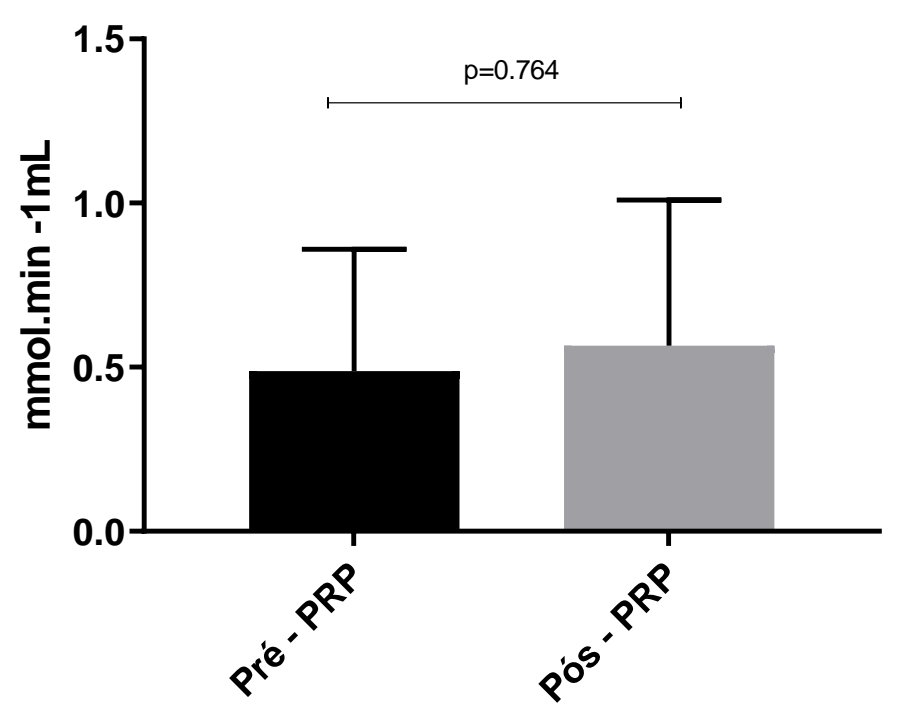

Legenda: Pré-PRP: pré protocolo de reabilitação pulmonar. Pós-PRP: pós protocolo de reabilitação pulmonar. Valores expressos em média \pm Desvio Padrão (DP).

Fonte: Wu SV, et al., 2021. 


\section{DISCUSSÃO}

Os resultados encontrados no estudo demonstram que o protocolo de reabilitação pulmonar foi capaz de reduzir a expressão sérica da creatina quinase total (CK-NAC) e da creatina quinase isoenzima MB (CK-MB) de pacientes com DPOC, no entanto não foi capaz de alterar as concentrações de peroxidação lipídica e da concentração da enzima antioxidante catalase. A Doença Pulmonar Obstrutiva Crônica (DPOC) constitui a quarta causa mais frequente de mortalidade do mundo, e estima-se que, até 2020 , ela seja a terceira principal causa de morte (KAPOOR S e GONZÁLEZ-GARCÍA M, 2014).

Segundo a BMJ- Best Practice (2020), a DPOC é mais comum em idosos, principalmente acima de 65 anos de idade. Corroborando com esses dados a média de idades, em anos, dos participantes do estudo foi superior a 60 anos. Outros estudos mostram uma prevalência de DPOC indivíduos com 60 anos ou mais de $15,4 \%$, sendo a maior dentre as faixas etárias analisadas, similar à encontrada no estudo (ASSIS NL e SHIMOYA-BITTENCOURT W, 2014; KOBLIZEK V, et al., 2016).

Dentre as manifestações clínicas da DPOC, destaca-se a disfunção muscular que possui relação direta com o aumento da gravidade e evolução da doença, influenciando no desempenho do exercício, no aumento da dispneia, piora no estado de saúde, com resultante decréscimo da qualidade de vida (ROCHA RS et. al., 2018). Atualmente, sugere-se que o estresse oxidativo desempenha um papel importante na gênese, desenvolvimento e manutenção do quadro clínico da disfunção muscular na DPOC (TAVILANI H, et al., 2012).

O estresse oxidativo reflete o desequilíbrio da relação entre a geração dos radicais livres e os mecanismos antioxidantes. Esse desequilíbrio pode resultar em danos sérios a diferentes estruturas celulares, tais como, oxidação de proteínas, danos no DNA e lipídeos de membrana. Esse dano aos lipídeos configura a peroxidação lipídica (SILVA ALG, et al., 2013). A peroxidação lipídica é umas das características centrais do estresse oxidativo e pode ser avaliada por vários métodos incluindo a quantificação de produtos de peroxidação. A mensuração da peroxidação lipídica nos sistemas biológicos é um parâmetro importante para a avaliação do estresse oxidativo celular (MOURA MMMC, et al., 2012).

No presente estudo, os resultados mostram que não houve alteração nos níveis plasmáticos de peroxidação lipídica no plasma do sangue periférico de indivíduos com DPOC submetidos ao PRP. Em contraste a esse resultado, Mercken EM et al. (2005) demonstraram que pacientes com DPOC submetidos a um programa de reabilitação pulmonar, de oito semanas, apresentaram diminuição dos níveis de peroxidação lipídica.

Outro importante marcador de estresse oxidativo é a quantificação da concentração do sistema antioxidante enzimático. Em sistemas aeróbicos é essencial o equilíbrio entre espécies reativas ao oxigênio e o sistema de defesa antioxidante (CAMPOS MTG e LEME FOP, 2018). Um aumento na produção de espécies reativas ao oxigênio e nitrogênio (ERON) ou uma pobre defesa antioxidante pode levar a um dano progressivo na célula com declínio na sua função. Para proteger-se, a célula possui um sistema de defesa que inclui antioxidantes enzimáticos e não enzimáticos. Juntos, o sistema antioxidante enzimático e não enzimático compõem a capacidade antioxidante total (HALLIWEL B, 2009).

Nesse contexto, pacientes com DPOC apresentam uma diminuição da capacidade antioxidante devido à exposição crônica ao estresse oxidativo, proveniente especialmente do tabagismo, principal fator de risco para desencadeá-la. Esse fato pode deixá-los mais suscetíveis a produção e ações danosas de radicais livres, porém com a prática de exercício físico observa-se uma melhora na capacidade antioxidante do organismo desses indivíduos (GONÇALVES APFG e COUTO VF, 2021).

No presente estudo verificou-se a capacidade antioxidante enzimática por meio da mensuração das concentrações da enzima catalase. Em relação aos níveis da enzima catalase, os resultados deste estudo mostram que as concentrações desse biomarcador não foram estatisticamente significantes quando comparadas antes e após o PRP em pacientes com DPOC. O exercício físico realizado de forma regular e de intensidade leve a moderada em PRP para pacientes com DPOC mostram-se eficazes na redução do estresse oxidativo por aumentar a capacidade antioxidante enzimática (ROCHA RS, et al., 2019). 
O estresse oxidativo é um dos principais responsáveis pelo mecanismo sistêmico da DPOC sendo o causador da disfunção muscular, levando ao aumento sistêmico da proteína creatina quinase (LOTTERMANN PC, et al., 2017). Considera-se que a liberação desta enzima para os vasos sanguíneos refletiria alterações importantes ocorridas na estrutura das membranas da fibra muscular (NUNES E e CURTY VM, 2012). Para avaliação da disfunção muscular em nível bioquímico, o presente estudo optou pela análise da CK, uma das proteínas mais estudadas e analisadas na literatura para análise do dano muscular (SOUZA EC, et al., 2016).

Estudos que quantificam a expressão de CK após protocolos de treinamento físicos em DPOC. Demonstraram que o protocolo de exercícios propostos foi capaz de diminuir os níveis de CK em paciente com DPOC e esses valores reduzidos se compararam aos valores basais de CK de indivíduos saudáveis (SOUZA EC, et al., 2016). Corroborando dessa forma, com os achados no presente estudo, os quais demonstram uma redução nos níveis da CK-NAC e CK-MAB após o PRP em pacientes com DPOC.

Outro estudo realizado com 60 pacientes com DPOC divididos em dois grupos, um treinava caminhada nórdica e outra caminhada livre, durante 3 meses obteve-se como resultado que o treinamento da caminhada nórdica na melhoria da saúde e condicionamento físico semelhante ao do presente estudo, os indivíduos obtiveram resultados significativos na redução dos níveis séricos de $\mathrm{CK}$, demonstrando que ocorreu uma redução nos níveis de desgaste muscular dos pacientes (ADOLFO JR, et al., 2019).

Nesse contexto, a PRP configura-se como um tratamento não farmacológico fundamental para minimizar os efeitos da DPOC (SOPTERJ, 2018). É indispensável frisar que o treinamento físico promove, melhora nos efeitos sistêmicos, na tolerância ao exercício, na redução do índice de dispneia e na melhora da capacidade funcional, minimizando, portanto, as complicações provenientes da doença, permitindo aos pacientes viver uma vida com menos restrições (WIJKSTRA PJ, 2011; MACHADO FLR, et al., 2011).

Diante do exposto, os autores elucidam a necessidade de insistir na investigação, acerca do comportamento dos parâmetros bioquímicos com maior tamanho amostral, a condução de um grupo controle para análises de comparabilidade, assim como estratificar a amostra quanto aos graus de disfunção pulmonar identificados pela espirometria.

\section{CONCLUSÃO}

Em conclusão, o presente estudo demonstrou que o programa de reabilitação pulmonar reduziu os níveis de dano muscular em paciente com DPOC, expresso não só pela análise bioquímica, mas também pela maior tolerância ao exercício. Contudo, o protocolo não alterou os níveis da peroxidação lipídica e da atividade antioxidante enzimática. Assim, torna-se necessário mais estudos com números amostrais maiores para a identificação dos aspectos bioquímicos que envolvem essa doença.

\section{AGRADECIMENTOS E FINANCIAMENTO}

Essa pesquisa foi financiada pelo projeto de iniciação científica do pelo Conselho Nacional de Desenvolvimento Científico e Tecnológico (CNPQ). Nossos singelos agradecimentos ao Laboratório de Morfofisiologia Aplicada à Saúde da Universidade do Estado do Pará, pelos materiais, equipamentos e espaço necessários para o desenvolvimento desta pesquisa; ao Mário Augusto Costa Prazeres e laboratório de Análises Clínicas na Universidade do Estado do Pará pelo auxílio, equipamento, espaço disponibilizado.

\section{REFERÊNCIAS}

1. ADOLFO JR, et al. Diferentes intensidades de exercício físico e a capacidade funcional na DPOC: revisão sistemática e meta-análise. Jornal Brasileiro de Pneumologia, 2019; 45(6): e20180011.

2. AEBI H. Catalase in vitro. Methods Enzymol. 1984; 105:121-6. doi: 10.1016/s0076-6879(84)05016-3. PMID: 6727660.

3. ASSIS NL, SHIMOYA-BITTENCOURT W. Perfil Epidemiológico dos Pacientes do Ambulatório de Pneumologia do Hospital Universitário Júlio Muller. CONNECTION LINE-REVISTA ELETRÔNICA DO UNIVAG, 2014; (7).

4. BMJ. Best Practice. Doença Pulmonar Obstrutiva Crônica: epidemiologia. Acesso em: 24 de jun. de 2020.

5. CAMPOS HS. Doença Pulmonar Obstrutiva Crônica: mais do que apenas uma doença pulmonar. Boletim de Pneumologia Sanitária, 2006; 14(1), 27-32. 
6. CAMPOS MTG, LEME FOP. Estresse oxidativo: fisiopatogenia e diagnóstico laboratorial. PUBVET (LONDRINA), 2018; 12: 1-8.

7. CAVALCANTE AGM, BRUIN PFC. O papel do estresse oxidativo na DPOC: conceitos atuais e perspectivas. Jornal Brasileiro de Pneumologia [online], 2009; 35(12): 1227-1237, ISSN 1806-3756.

8. DHAKAL N, et al. Oxidative stress and nutritional status in chronic obstructive pulmonary disease. Journal of Clinical and Diagnostic Research: JCDR, 2015; 9(2): BC01.

9. FIORENTINO G, et al. Exercise and Chronic Obstructive Pulmonary Disease (COPD). In: Physical Exercise for Human Health. Springer, Singapore, 2020; 1228: 355-368.

10. FISCHER BM, et al. Pathogenic triad in COPD: oxidative stress, protease-antiprotease imbalance, and inflammation. International Journal of Chronic Obstructive Pulmonary Disease, 2011; 6: 413-421.

11. GONÇALVES APFG, COUTO VF. Efeitos da atividade física sobre a doença pulmonar obstrutiva crônica: uma revisão de literatura. Lecturas: Educación Física y Deportes, 2021; 25(274): 196-208.

12. HALLIWELL B. The wanderings of a free radical. Free Radical Biology \& Medicine, 2009; 46: 531-542.

13. KAPOOR S, GONZÁLEZ-GARCÍA M. Incidência de embolia pulmonar durante exacerbação da DPOC. Jornal Brasileiro de Pneumologia, 2014; 40(1): 96-97.

14. KOBLIZEK V, et al. Diagnosing COPD: advances in training and practice - a systematic review. Advances In Medical Education And Practice, 2016; 7 (1): 219-231.

15. LAIZO A. Doença pulmonar obstrutiva crónica - Uma revisão. Revista Portuguesa de Pneumologia [online], 2009; 5(6): 1157-1166.

16. LOTTERMANN PC, et al. Programas de exercício físico para pessoas com DPOC: uma revisão sistemática. Arq. ciências saúde UNIPAR , 2017; 21(1): 65-75.

17. MACHADO FLR, et al. Efeitos do exercício físico combinado na dispnéia, capacidade funcional e qualidade de vida de pacientes com DPOC em uma clínica privada. ASSOBRAFIR Ciência, 2011; 2(2):19-28.

18. MERCK SHARP AND DOHME. Wise RA MSD. Doença Pulmonar Obstrutiva Crônica (DPOC). Manual MSD- versão para profissionais de saúde, 2018.

19. MERCKEN EM, et al. Rehabilitation decreases exercise-induced oxidative stress in chronic obstructive pulmonary disease. American Journal of Respiratory and Critical Care Medicine, 2005; 172(8): 994-1001.

20. MOURA MMMC, et al. O papel dos biomarcadores na doença pulmonar obstrutiva crônica (Tese de Doutorado). Faculdade de Medicina da Universidade de Coimbra, 2012.

21. NUNES E, CURTY VM. Variação da atividade sérica de creatina kinase induzida pelo treinamento em circuito. Revista Brasileira de Prescrição e Fisiologia do Exercício, 2012; 35 (6): 465-471.

22. RABAHI MF. Epidemiologia da DPOC: Enfrentando Desafios. Pulmão - Rio de Janeiro, 2013; 22 (2): 4-8.

23. ROCHA RS, et al. A influência de um programa de reabilitação pulmonar na descontinuidade da matriz extracelular, no estado de saúde e na resposta ao exercício em pacientes com DPOC. Revista Brasileira de Ciência e Movimento. 2018; 26 (4): 67-75.

24. ROCHA RS, et al. A influência de um programa de reabilitação pulmonar na descontinuidade da matriz extracelular, no estado de saúde e na resposta ao exercício em pacientes com DPOC. Revista Brasileira de Ciência e Movimento, 2019; 26 (4): 67-75.

25. RUGBJERG M, et al. Effectiveness of pulmonary rehabilitation in COPD with mild symptoms: a systematic review with meta-analyses. International journal of chronic obstructive pulmonary disease, 2015; 10: 791.

26. SEVERO VG, RECH VV. Reabilitação pulmonar: treinamento de membros superiores em pacientes com DPOC; uma revisão. Fisioterapia e pesquisa, $2006 ; 13$ (1): 44-52.

27. SILVA ALG, et al. DNA Damage and Oxidative Stress in Patients with Chronic Obstructive Pulmonary Disease. The Open Biomarkers Journal, 2013; 6: 1-8.

28. SILVA BS, et al. Resistance Training With Elastic Tubing Improves Muscle Strength, Exercise Capacity, and PostExercise Creatine Kinase Clearance in Subjects With COPD. Respiratory care, 2015; 64 (7): 835-843.

29. SILVA BSA, et al. Inflammatory and Metabolic Responses to Different Resistance Training on Chronic Obstructive Pulmonary Disease: A Randomized Control Trial. Frontiers in Physiology, 2018; 9 (1): 262.

30. SILVA BSDA. Avaliação da força, estresse muscular e marcadores inflamatórios de indivíduos com doença pulmonar obstrutiva crônica submetidos a treinamento resistido com tubos elásticos. (Dissertação de Mestrado - Faculdade de Ciências e Tecnologia). Presidente Prudente (SP): Universidade Estadual Paulista; 2015.

31. SKÓRKOWSKA-TELICHOWSKA K, et al. Nordic walking in the second half of life. Aging clinical and experimental research, 2016; 28(6): 1035-1046.

32. SOPTERJ. Sociedade de Pneumologia e Tisiologia do Estado do Rio de Janeiro. Protocolo de diagnósticos e tratamento de Doença Pulmonar Obstrutiva Crônica da Sociedade do Estado do Rio de Janeiro, 2018.

33. SOUSA AS, et al. Exercício físico no tratamento de pacientes com doença pulmonar obstrutiva crônica: quais os benefícios?. Revista Brasileira de Fisiologia do Exercício, 2018; 17(1): 64-70.

34. SOUZA EC, et al. Creatine Kinase level in patients with chronic obstructive pulmonary disease treated with hidrotherapy. Revista Interdisciplinar de Estudos em Saúde, 2016; 5(1): 95-104.

35. TAVILANI H, et al. Oxidative Stress in COPD Patients, Smokers, and Non-smokers. Respir Care, 2012; 57(12): 20902094.

36. TROMM CB, et al. A diferença de idade é um fator determinante na modulação do estresse oxidativo muscular induzido pelo exercício agudo, 2018; 40(3): 273-280.

37. WIJKSTRA PJ, WEMPE JB. Novas ferramentas na reabilitação pulmonar. European Respiratory Journal, 2011; 38(6): 1468- 1474.

38. ZAU JFT. Avaliação do estresse oxidativo em pacientes coronariopatas revascularizados submetidos à reabilitação cardíaca. Dissertação (mestrado) - Universidade Federal do Rio de Janeiro, Rio de Janeiro, 2016.

39. ZOPPI D, FILHO ABA. Doença Pulmonar Obstrutiva Crônica- exacerbação aguda na sala de urgência. Revista qualidade HC, Ribeirão Preto: US, 2018. 